\title{
Strates
}

STRATES Matériaux pour la recherche en sciences sociales

15 | 2008

Union européenne - Voisinages. La quête d'une intégration régionale

\section{L'Union européenne et ses voisinages. Organiser la relation pour construire une région mondiale}

Yann Richard

\section{(2) OpenEdition}

Journals

Édition électronique

URL : http://journals.openedition.org/strates/6523

DOI : $10.4000 /$ strates.6523

ISSN : $1777-5442$

Éditeur

Laboratoire Ladyss

Édition imprimée

Date de publication : 1 janvier 2008

Pagination : 9-22

ISSN : 0768-8067

Référence électronique

Yann Richard, «L'Union européenne et ses voisinages. Organiser la relation pour construire une région mondiale », Strates [En ligne], 15 | 2008, mis en ligne le 25 janvier 2013, consulté le 08 septembre

2020. URL : http://journals.openedition.org/strates/6523; DOI : https://doi.org/10.4000/strates.6523

Ce document a été généré automatiquement le 8 septembre 2020

Tous droits réservés 


\title{
L'Union européenne et ses
} voisinages. Organiser la relation pour construire une région mondiale

\author{
Yann Richard
}

1 L'Union européenne (UE) joue les premiers rôles dans la globalisation. Certains indicateurs classiques viennent le rappeler régulièrement au gré de nombreuses publications. Son poids économique est considérable. La part consolidée de l'actuelle UE à 27 dans le produit mondial brut oscille autour de $30 \%$ depuis les années 1980, avec un maximum à $33 \%$ en 1993 et un minimum de $26 \%$ en 2000. Si on lui ajoute les pays de l'AELE (Association européenne de libre-échange) dont la Suisse, l'espace économique européen pèse même 1,5 à 2 points de plus.

On en viendrait à la même conclusion si l'on s'appuyait sur d'autres indicateurs macroéconomiques, comme la part de l'UE dans le commerce mondial des biens et services ou plus encore dans le total mondial des investissements internationaux sortants et entrants. Déjà l'UE à 15 réalisait $17 \%$ du commerce mondial de marchandises en 2000 en excluant du calcul les échanges internes entre pays membres (contre $16 \%$ pour les États-Unis). En ce qui concerne les Investissements directs étrangers (IDE), la domination de l'UE est encore plus nette que pour le commerce international ou pour la production de richesse. Les pays qui constituent l'actuelle UE à 27 absorbaient autour de $40 \%$ des flux annuels entrants dans les années 1970 et autour de $50 \%$ dans les années 2000 avec un pic à $58 \%$ en 2003 (Cnuced, Banque mondiale). À titre de comparaison, l'ensemble États-Unis/Canada absorbait environ $30 \%$ des IDE entrants dans les années 1970 et moins de $20 \%$ dans les années 2000. L'Asie orientale vient loin derrière malgré la montée en puissance de la Chine.

3 L'UE s'insère dans la mondialisation de plusieurs façons. Elle constitue d'abord un des trois pôles de la Triade et ses plus grandes villes font bonne figure dans ce qu'on appelle l'archipel métropolitain mondial (Dollfus, 1994 et 1999 ; Veltz, 1996). Dans ce 
que Wallerstein a défini comme le système monde (Wallerstein, 1979, 2002 et 2006), elle joue le rôle d'un centre qui exerce une influence évidente sur certaines régions (Didelon, Grasland, Richard, 2008). Cette influence se donne à voir à travers de nombreux indicateurs qui montrent que l'Union se projette puissamment vers l'extérieur: commerce, investissements, migrations internationales, aide au développement... L'UE est par exemple le premier partenaire commercial de nombreux pays situés principalement en Afrique, autour de la Méditerranée, dans la CEI... Sa prééminence est plus nette encore dans de très nombreux pays en ce qui concerne les investissements directs. Inversement, le territoire de l'UE est pénétré de toutes parts par la mondialisation, qui contribue à le façonner et à y dégager des lignes de force et des contrastes entre régions riches et pauvres, entre métropoles bien connectées et espaces moins accessibles.

\section{L'UE : un poids lourd en recul relatif dans la globalisation}

4 La place de l'UE dans la mondialisation est éminente mais elle n'est pas assurée une fois pour toutes. D'une part, si l'on prend en considération quelques indicateurs que l'on associe couramment à la puissance économique, comme la surface agricole utile, la population ou le PIB (ce dernier exprimé en parité de pouvoir d'achat), on constate qu'aucun État membre ne figure parmi les dix premiers pays dans le monde à l'exception de l'Allemagne pour le PIB. D'autre part, si on considère la part de l'UE dans le total mondial pour quelques-uns des indicateurs mentionnés plus haut, on constate qu'elle tend à reculer depuis plusieurs décennies. Sa part dans les exportations mondiales de biens et de services baisse sensiblement, passant de $40 \%$ dans les années 1980 à $35 \%$ dans les années 2000. La baisse de son poids démographique n'est pas une surprise (cf. figure 1). Mais sa part décroissante dans les exportations, bien qu'elle ne révèle pas un appauvrissement, est le signe que la croissance économique tend à se déplacer vers d'autres régions du monde. Sans se poser en déclinologue - ce n'est le propos de cet ouvrage -, ces quelques signes posent de nombreuses questions et soulignent quelques réalités qu'il n'est plus possible d'ignorer : le poids relatif des pays membres de l'UE dans le monde baisse ; jusqu'à aujourd'hui, l'UE n'a pu maintenir ce poids relatif (démographique et économique) dans le monde qu'à la faveur de ses élargissements successifs (Beckouche, Grasland, Didelon, Richard, 2008; Didelon et Grasland, 2007). En d'autres termes, économiquement et démographiquement (cf. figure 1), l'UE à 27 ne pèse guère plus lourd dans le monde de 2008 que la Communauté économique européenne à 6 dans le monde de l'après-guerre. 
Figure 1. Évolution du poids démographique de la Communauté européenne dans la population mondiale au fil de ses élargissements.

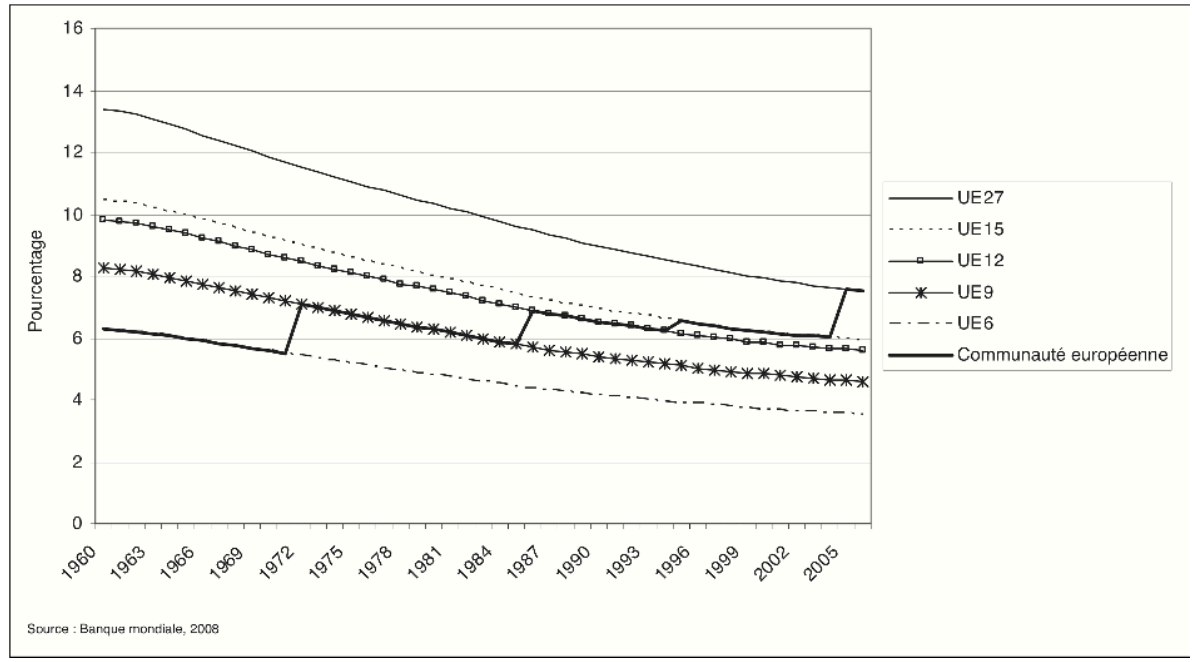

Pour autant, le salut est-il dans l'élargissement sans fin? Ce processus approche de son terme : la Turquie est candidate officielle et les pays des Balkans occidentaux vont adhérer un à un. Mais ensuite ? Peut-être quelques pays de l'ex-URSS, l'Ukraine, la Moldavie? Mais rien n'est moins sûr car les conditions de futurs élargissements importants ne sont pas réunies. Il faudrait songer à modifier encore une fois certains dispositifs institutionnels déjà réformés avec beaucoup de difficulté (traité de Nice, échec du premier traité constitutionnel); les opinions publiques européennes n'y sont pas favorables; la situation économique et financière, donc budgétaire, de l'Union ne s'y prête pas; les éventuels nouveaux membres sont pauvres, ce qui serait facteur supplémentaire de fragilisation...

En bref, l'Union est face à deux impératifs liés. Elle est contrainte de trouver les moyens de continuer à peser dans une globalisation en manque de régulation. Pour ce faire, elle doit améliorer la qualité de ses relations avec ses voisins, en faisant preuve d'imagination et d'esprit de partenariat, afin de se placer au centre d'un ensemble régional consolidé vaste et visible, sans être contrainte de poursuivre un processus d'élargissement institutionnel dont elle n'a pas les moyens. Ce contexte particulier invite à porter un autre regard vers ce qui se passe au-delà des frontières de l'UE, surtout vers un au-delà tout proche qu'on désigne par le terme englobant de "voisinages». Renforcer les relations de toutes natures avec ces pays serait-il une réponse appropriée aux évolutions qu'on vient de souligner?

\section{Les voisinages entre intégration et mise à distance}

Les Européens accordent de plus en plus d'attention à ces "voisinages " depuis les années 1990, et davantage encore depuis le début des années 2000. Cet intérêt s'explique facilement. L'ordre géopolitique européen a changé avec l'effondrement de l'Union soviétique; les Peco se sont tournés vers l'Ouest; les Balkans occidentaux doivent être stabilisés ; plusieurs pays ont fait acte de candidature (Moldavie, Ukraine, Géorgie) ou sont candidats officiels (Croatie, Macédoine, Turquie); l'instabilité politique régionale inquiète; la pression migratoire se fait sentir dans le voisinage géographique sud et oriental; l'approvisionnement en ressources énergétique est au 
sommet de l'agenda politique européen... La prise de conscience a été stimulée par la Commission européenne en plusieurs étapes : conseil européen de Copenhague en 2002, publication de la communication «Voisinages » en 2003, lancement des programmes de voisinage en 2004... Peu à peu, l'UE prend acte de plusieurs réalités qui s'imposent à elle : les relations entre elle et ses voisinages sont déjà intenses, dans les deux sens ; dans certains cas, on peut même parler d'interactions, tant il est clair que ce qui se passe en dehors a un impact sur ce qui se passe au-dedans et inversement (Didelon \& Grasland, 2007).

8 La géographie des échanges commerciaux montre que les pays qui constituent le voisinage géographique de l'actuelle UE à 27 pèsent de plus en plus dans son commerce extérieur : ils absorbaient moins de $4 \%$ de ses exportations en 1992 mais presque $7 \%$ en 2006 ; leur part dans les importations de l'UE, tirée il est vrai par l'augmentation du prix des matières premières énergétiques, est passée de 3,5\% à $7 \%$ pendant la même période. En même temps, le commerce interne à l'UE tend à reculer. Ces deux tendances combinées révèlent un élargissement de l'assise territoriale de l'ensemble géoéconomique européen. En d'autres termes, l'UE est fortement impliquée dans le processus de régionalisation de l'économie mondiale (Frankel, 1998 ; Siroën, 2000) mais en associant de plus en plus les pays proches d'elle. Dans ce processus qui se joue à une échelle macrorégionale, il existe des nuances notables selon les pays. Par exemple, les échanges commerciaux stagnent avec les pays de la région méditerranéenne et ils augmentent dans les deux sens avec ceux de la CEI. Inversement, les investissements directs, pourtant nécessaires à certains pays voisins mieux pourvus en main-d'œuvre qu'en capitaux et en technologies, continuent de se heurter à un fort effet frontière. Les entreprises françaises envoient par exemple chaque année moins de $1 \%$ de leurs investissements vers les économies d'Afrique. À titre de comparaison, près de $18 \% \mathrm{du}$ stock cumulé des investissements faits par les entreprises japonaises dans le monde en 2003 se trouvaient en Asie orientale. D'une façon générale, les entreprises des pays membres de l'UE investissent très peu dans les pays voisins (cf. figure 2). Les Peco (pays d'Europe centrale et orientale), qui étaient les voisins de l'UE jusqu'en 2004, ont été une exception de ce point de vue: après la dissolution du CAEM (Conseil d'assistance économique mutuel) et du bloc soviétique, leur croissance rapide a reposé entre autres sur une aide au développement massive et sur un afflux considérable d'IDE venus essentiellement de l'UE. Ce n'est pas encore le cas pour les pays riverains de la Méditerranée ni pour les anciennes républiques soviétiques. 
Figure 2. Destination des stocks d'IDE sortant de l'UE 15 en 2004.

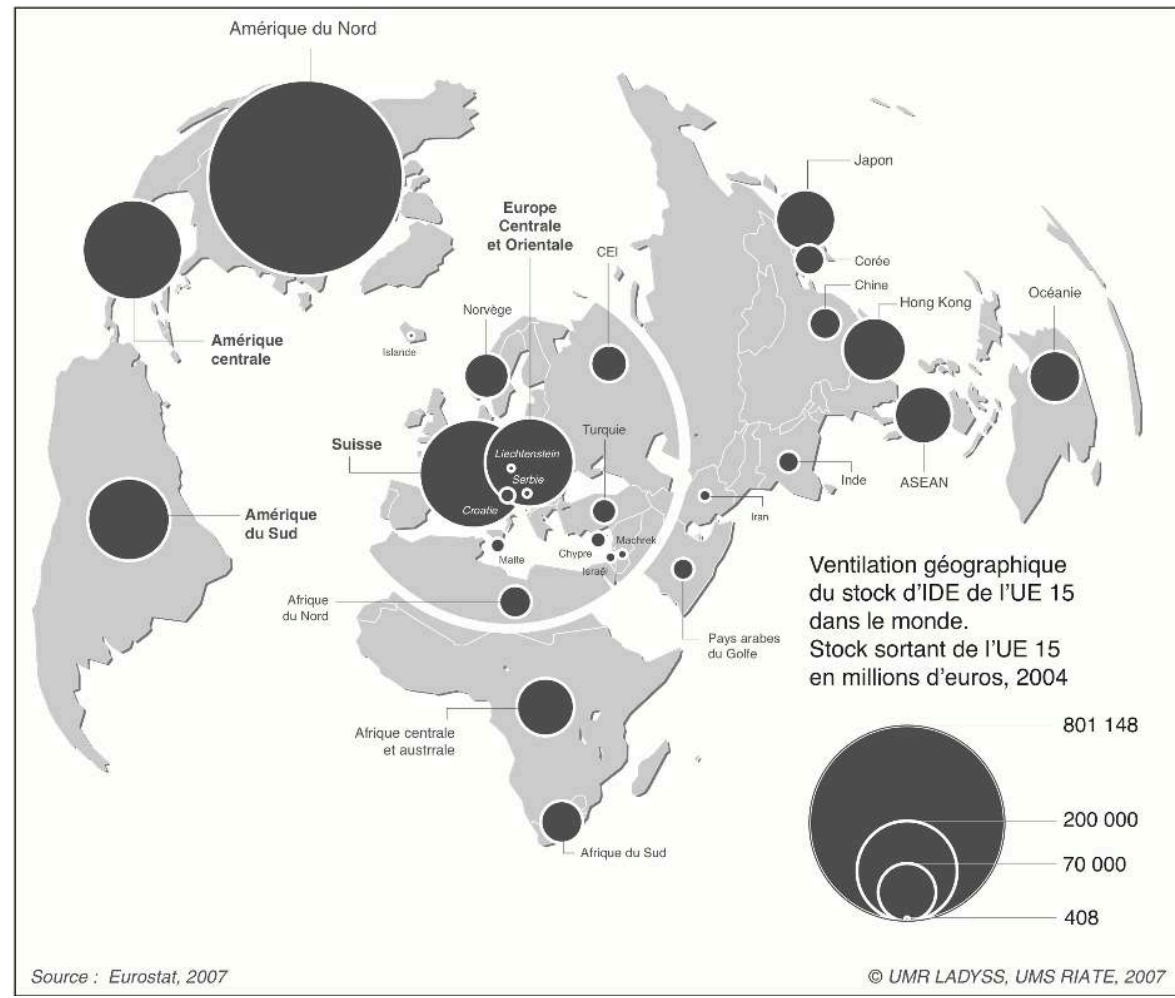

9 Les relations fortes entre l'UE et la plupart des pays proches, tant pour le commerce que pour d'autres types de flux (touristes, migrants internationaux, aide au développement...), évoquent un système de type centre périphérie. Sur cette base, on peut considérer qu'il existe une vaste région qu'on pourrait baptiser « Euroméditerranée » ou « grande région européenne » ou « Europe élargie »... Elle est composée de l'UE et de ses «voisinages». Ce terme recouvre une réalité complexe qu'il faut définir dès maintenant pour lever certaines ambiguïtés. Le conseil européen de Copenhague (12 et 13 décembre 2002) a lancé le terme "voisins" pour désigner l'ensemble des pays proches de l'UE, sans qu'ils soient nécessairement éligibles à ce qui allait devenir la politique européenne de voisinage ${ }^{1}$. Par la suite, les documents officiels de l'UE montrent un certain flottement puisqu'ils passent insensiblement du mot « voisin » ou « voisins » à « voisinage » et « voisinages "². À partir de 2003, il semble que l'expression «voisinage» tienne la corde ${ }^{3}$. Dans ce dernier cas, ce sont les voisins du sud et ceux de l'est qui sont pris en considération en même temps, reprenant ainsi l'acception large proposée par Romano Prodi dans une célèbre communication publiée la même année sur l'Europe élargie ${ }^{4}$. La notion de "voisinage " au singulier s'est peu à peu imposée et s'est stabilisée dans son contenu en excluant la Russie (dont le statut est particulier depuis 2003 avec la création de 4 espaces communs « Russie - UE » définis par 4 domaines de coopération) et en incluant finalement les pays du Caucase (Arménie, Azerbaïdjan, Géorgie). Il s'agit ni plus ni moins des pays éligibles à la politique européenne de voisinage ${ }^{5}$. Mais l'usage du mot au singulier et sa définition géographique officielle sont trop restrictifs car ils font mine d'ignorer que l'Union entretient aussi des relations très fortes avec des pays proches d'elle mais non éligibles à la politique de voisinage, parce qu'ils ne partagent pas avec elle de frontière commune ou parce qu'ils sont liés à elle par d'autres types de relations contractuelles. Pour saisir les limites imprécises et mouvantes du vaste ensemble géographique qui 
constitue l'objet central des articles qui suivent, la "région européenne élargie ", il vaut mieux prendre en compte l'ensemble des pays proches géographiquement de l'UE à 27 et liés à elle par des relations fortes, sur une base contractuelle ou non.

\section{Le voisinage ou les voisinages?}

10 Le mot " voisinage » pris au singulier est trompeur et inapproprié. Il vaut mieux parler de «voisinages » au pluriel car cette vaste auréole est composée par des pays dont les niveaux de développement et surtout les statuts, eu égard à leur relation avec l'UE, sont variés. Les plus nombreux sont les pays éligibles à la politique de voisinage. Ils forment une auréole qui va du Maroc à la Biélorussie, en passant par les rives de la Méditerranée, le Caucase et l'Europe orientale.

11 Avant 2007, l'ensemble des pays de l'ex-URSS étaient éligibles à l'un des programmes européens d'aide au développement de l'UE: le programme Tacis. Une réforme intervenue le 1^janvier 2007 a créé, entre autres, un Instrument européen de voisinage et de partenariat (IEVP) qui permettra de financer la Politique européenne de voisinage. Cet instrument concentre l'aide européenne sur les pays de la CEI occidentale et sur les pays du Caucase, en plus des pays méditerranéens. L'Asie centrale est donc laissée de côté. Pour autant, cette région est l'objet d'une attention particulière en Europe car elle possède des ressources qui pourraient contribuer à diversifier les sources et les routes d'approvisionnement de l'Union en gaz et en pétrole. Il y a là une sorte de «voisinage potentiel » où la visibilité de l'UE est encore faible malgré un regain récent d'activité diplomatique de la Commission européenne ${ }^{6}$.

12 Les pays des Balkans occidentaux sont aussi des voisins géographiques de l'Union, formant un ensemble entièrement entouré par des pays membres. Mais ils ne sont pas éligibles à la Politique de voisinage. Leur relation avec l'UE se construit dans le cadre d'accords de stabilisation et d'association conçus pour mener à leur adhésion. Ils bénéficient partiellement, depuis le $1^{\text {er }}$ janvier 2007, d'un nouvel instrument financier censé donner vie à ces accords : l'instrument d'aide de pré-adhésion.

Les pays « candidats officiels » (Turquie, Croatie, Macédoine) forment un autre groupe. Ils bénéficient de l'instrument d'aide de pré-adhésion, mais dans sa version complète. Enfin, la Russie est dans une situation particulière. Elle peut recevoir des fonds européens issus de l'Instrument financier de la politique de voisinage (l'IEVP), mais elle n'est pas un «voisin » sur le plan institutionnel. Ses relations avec l'UE se construisent sur la base d'autres décisions politiques, il est vrai peu suivies d'effets : quatre espaces communs créés en 2003.

Le tableau parait simple. Il juxtapose les « voisins » au sens institutionnel (bénéficiaires de l'IEVP), les candidats officiels, les candidats potentiels des Balkans occidentaux, la Russie. Dans les faits, il n'en est rien. Les relations institutionnelles de l'Union avec « ses voisinages » sont beaucoup plus diversifiées et complexes. Par ailleurs, la situation est en évolution constante. Surtout, elle repose dans certains cas sur des documents peu contraignants et peu efficaces, voire obsolètes (ce qui est le cas de l'accord de partenariat et de coopération entre l'UE et la Russie ; Bordatchev, 2007 ; Gomart, 2007). L'Union s'est engagée de facto dans la construction de relations de voisinage à la carte, bâtissant autour d'elle une enveloppe régionale de plus en plus bigarrée. La Turquie, par exemple, se distingue des autres candidats officiels : elle est membre de l'union douanière européenne depuis déjà plus de 10 ans. La Biélorussie n'est que 
théoriquement éligible à la Politique de voisinage et n'en profitera pas pleinement tant qu'elle ne s'engagera pas dans un processus de démocratisation. De même pour la Syrie et la Libye qui, pour des raisons un peu différentes, n'ont qu'un statut d'observateur. L'Ukraine multiplie quant à elle les partenariats et les coopérations de toutes sortes avec Bruxelles, donnant mille gages de sa bonne volonté pour que l'Union prenne au sérieux son projet d'adhésion. Elle souhaite l'établissement d'un partenariat renforcé. L'accord de partenariat qui la lie à l'Union, dans sa version reconduite pour 2008, comporte des clauses qui la différencient déjà des autres "voisins". Enfin, la négociation sur l'ouverture d'une zone de libre-échange avec l'UE est en cours. Le Maroc s'engage lui aussi dans la négociation d'un tel partenariat renforcé. Ce pays, ainsi que l'Égypte et la Tunisie (bientôt Israël), a déjà commencé des négociations bilatérales avec Bruxelles pour libéraliser les échanges de services. Enfin, la Moldavie a obtenu récemment de l'Union certaines préférences commerciales, tandis que la Géorgie et l'Arménie souhaitent l'instauration d'une zone de libre-échange... Autant de pays, autant de statuts, autant de réglementations... Sans oublier ces voisins riches que sont les pays de l'AELE dont certains sont très proches de l'Union sans en être membres à part entière, soit qu'ils font ou vont faire prochainement partie de l'espace Schengen (Norvège, Islande, Suisse), soit qu'ils sont membres de l'Espace économique européen (UE 27 + Norvège + Islande + Liechtenstein) et donc membres du marché commun...

Au-delà de cette mosaïque de particularités institutionnelles qui se surimposent à des relations fonctionnelles elles-mêmes très diverses dans leur intensité et leur nature (commerce, migrations, tourisme...), les voisinages forment des sous-ensembles différenciés. Les pays riverains de la Méditerranée n'ont pas les mêmes caractères que les voisins orientaux membres de la CEI, les Balkans occidentaux ou la Turquie. Les pays méditerranéens sont des pays en développement. Certains sont engagés dans un processus de convergence avec l'UE, ce que révèle par exemple l'évolution des indicateurs démographiques et de l'indice de développement humain. Mais leur situation économique ne montre pas toujours les signes d'une amélioration robuste et durable. Les voisins de l'est offrent un visage différent. Ce sont des pays issus de l'URSS où l'héritage soviétique n'est pas uniment négatif : structure économique relativement diversifiée, main-d'œuvre bien formée, tradition industrielle, infrastructures plus denses et de meilleure qualité que dans beaucoup de pays en développement, présence d'une recherche et d'une maîtrise technologique reconnue dans certains domaines, présence de capital (la Russie est émettrice nette d'investissements internationaux)... Leur situation démographique est très différente de celle des pays méditerranéens, avec des soldes naturels fortement négatifs et une des fécondités les plus basses du monde. Les pays du voisinage sud, même lorsqu'ils convergent avec l'Europe sur ce plan, ont une natalité plus élevée à cause d'une structure par âge plus jeune. En outre, les pays du voisinage oriental forment toujours un ensemble macrorégional fortement intégré sur le plan économique et commercial (Richard, Zanin, 2007) où l'influence de la Russie reste forte. Par ailleurs, leurs relations avec les Peco demeurent intenses (Broadmann, 2005). Plusieurs décennies d'appartenance à l'ensemble soviétique et au Conseil d'Assistance économique mutuel ne s'effacent pas si vite (Dressler, 2008). Inversement, les pays riverains de la Méditerranée ne forment pas un ensemble intégré : ils sont confrontés à des problèmes communs mais ils sont désunis, séparés pour certains par des fractures politiques difficiles à réduire. La question de la relation entre l'UE et ses voisins ne se pose donc pas partout dans les mêmes termes. 


\section{Passer de la régionalisation au régionalisme}

16 Ce contexte particulier rend difficile le passage d'une intégration régionale essentiellement fonctionnelle (économie, migrations économiques, tourisme...), plutôt spontanée voire subie par les pays voisins, à une intégration plus poussée où le volet institutionnel trouverait davantage de place. Alors que d'évidentes complémentarités existent entre l'Union et ses voisinages, celle-là peine à passer de la régionalisation (avec une forte composante économique libérale) à un régionalisme Nord-Sud et OuestEst bien tempéré et non asymétrique dont les avantages pour les deux parties mériteraient pourtant d'être explorés (Beckouche, Grasland, Didelon, Richard, 2008). Le régionalisme se distingue de la régionalisation de l'économie. La seconde désigne l'augmentation des échanges de tous types entre des États situés dans la même région du monde. Elle a généralement un caractère «spontané ». Elle est portée par les firmes ou se donne à voir dans les pratiques individuelles ou collectives (tourisme). Le régionalisme désigne un rapprochement concerté entre plusieurs États proches les uns des autres qui signent des traités afin de réguler leurs relations à l'échelle régionale : il peut s'agir de traités de libre-échange, d'unions douanières, d'unions monétaires, de traités sur la protection réciproque des investissements, d'accords migratoires... À la régionalisation qui se présente comme un processus de facto, attestant de l'importance persistante de la proximité spatiale dans la géographie des échanges, le régionalisme ajoute une base juridique explicite et ouvre la voie à différentes formes de coopération ou de partenariat sur une autre base.

17 L'UE souhaite-t-elle vraiment franchir le pas du régionalisme avec ses voisinages? Certains indices montrent qu'une telle démarche régionaliste, pourtant explicite dans les discours, manque encore de substance dans les faits. Par ailleurs, la méthode utilisée par l'UE pour établir des relations plus institutionnelles avec les voisinages n'est pas toujours appropriée car elle est essentiellement bilatérale. Elle s'appuie principalement sur des plans d'action signés par la Commission européenne avec chaque pays partenaire. Une telle méthode se justifie dans certains cas. Elle est une façon de prendre en considération les caractères particuliers de chaque pays. Mais elle est inappropriée lorsqu'il s'agit de traiter des problèmes qui sont par essence transnationaux: lutte contre la pollution, gestion raisonnée des migrations internationales, approvisionnement en matières premières énergétiques, renforcement de la paix et de la stabilité régionale... Surtout, elle est dépassée par certaines évolutions. Est-il pertinent de multiplier des accords bilatéraux avec les pays de la CEI quand ces derniers avancent dans la construction d'une Communauté économique eurasiatique et même d'une Union douanière?

Pour l'heure, les tentatives pour mettre en œuvre une approche régionale et pour progresser vers une relation mieux régulée avec les voisinages n'ont pas eu beaucoup de résultats concrets (Philippart, 2003 ; Beckouche, 2008). L'échec est imputable autant à l'UE, qui essuie sur ce plan de nombreux reproches depuis longtemps (rappelons l'échec retentissant du 10. anniversaire du processus de Barcelone où seulement deux chefs d'États méditerranéens sont venus), qu'aux partenaires, qui sont eux-mêmes réticents à l'idée de renforcer leur partenariat avec l'UE. Qu'en sera-t-il du projet d'Union pour la Méditerranée, porté par la France et érigé au rang de projet européen par le conseil européen de mars 2008 ? Apportera-t-il une valeur ajoutée au poussif processus de Barcelone? Il est vrai que ce projet propose quelques innovations qui 
peuvent retenir l'attention des pays partenaires méditerranéens. L'Union sera animée par une présidence conjointe assurée par un pays du sud et un pays du nord de la Méditerranée. Elle sera dotée d'un secrétariat permanent. Celui-ci (situé au sud de la Méditerranée) assistera la présidence pour organiser tous les deux ans un sommet des pays concernés. Le sommet européen de juillet 2008 en dira davantage sur le contenu concret de cette nouvelle initiative qui vise à relancer un véritable partenariat à l'échelle régionale.

19 À l'est, certains partenaires de l'Union refusent eux aussi de s'engager trop loin dans le partenariat. La Russie rejette par principe l'idée du moindre transfert de souveraineté et résiste à s'engager dans des coopérations trop poussées, préférant une coopération méfiante à une intégration. Elle se perçoit davantage comme un centre dominant que comme une partie d'un ensemble qui la dépasserait et sur lequel elle n'aurait pas vraiment de prise (Gomart, 2007). Aussi voit-elle d'un mauvais œil les propositions européennes de coopération transnationale et craint l'émergence d'une

UE trop puissante à ses portes (Arbatova, 2007). Il est symptomatique qu'elle préfère de loin développer des relations bilatérales avec certains pays membres qu'avec l'Union dans son ensemble. On peut signaler à ce propos qu'il n'existe pas de direction chargée des relations avec l'UE au ministère russe des Affaires étrangères. D'une façon générale, la relation de l'UE avec les voisins ne se construit pas toujours comme un partenariat raisonné et négocié. Elle fonctionne davantage comme un jeu donnant donnant agrémenté de pressions diverses et de concessions en vue d'obtenir quelques avantages ponctuels en retour. La Russie, par exemple, a signé un accord de réadmission en obtenant en échange des facilités consulaires pour certains de ses ressortissants. L'Ukraine en a fait autant, en espérant que la perspective d'une adhésion à l'Union même sans date précise sera explicitement mentionnée dans la version rénovée de l'accord de partenariat et de coopération qu'elle négocie avec Bruxelles. En bref, du chemin reste encore à faire vers de réels partenariats, même si les communications de la Commission parlent souvent non pas d'une intégration à faire mais plutôt d'une "intégration à renforcer", comme si le processus était déjà lancé. Pure rhétorique destinée à rassurer les pays voisins et ne pas les laisser penser que l'Union souhaiterait les reléguer définitivement dans un voisinage sans espoir d'adhésion.

Théoriquement, l'ensemble constitué par l'UE à 27 et tous ses voisins pèse très lourd et est bien visible à l'échelle mondiale, même si son poids relatif tend à baisser. L'ensemble aurait représenté $40 \%$ du produit mondial brut en 1990 et $36 \%$ en 2006 ; dans le même temps, la part de l'Alena (l'Accord de libre-échange Nord-américain) est passée de $30 \%$ à $32 \%$ et celle de l'Asie de l'est et du sud-est est restée stable aux environ de $18 \%$ (avec un pic à $25 \%$ en 1995). La part de l'ensemble «UE - Voisinages » dans la production mondiale (PIB) baisse également mais elle reste très supérieure à celle des deux autres ensembles régionaux. Cette tendance à la baisse relative de l'ensemble «UE - Voisinages » dans le total mondial est à peu près générale, y compris pour la population. Par ailleurs, ces performances, tout de même flatteuses, ne sont que théoriques car elles reposent sur l'addition artificielle d'indicateurs nationaux. Cela dit, les chiffres mentionnés ci-dessus ont été calculés de la même façon pour l'Alena et l'Asie du Sud et du Sud-Est. La comparaison n'est donc pas totalement dénuée de sens. Peut-on pour autant parler d'une véritable région intégrée qui serait perçue en tant que telle par les autres pays du monde ? Si un minimum de contenu institutionnel et de relations contractuelles (portées par des acteurs publics, parapublics voire privés) ne 
vient pas donner corps à cet ensemble, l'Euroméditerranée ne sera jamais qu'une juxtaposition incertaine de pays sans orientation commune et sans personnalité. Elle continuera de juxtaposer un centre riche cerné par des pays plus pauvres où s'accumulent des problèmes sur lesquels l'influence de l'UE restera dangereusement limitée.

Cette situation n'est pas souhaitable si l'UE prétend atteindre certains objectifs vitaux à long terme : stabiliser l'environnement régional sur le plan politique, engager le cercle vertueux du développement social et économique dans les pays proches, pérenniser et sécuriser l'approvisionnement énergétique, favoriser le développement régional à toutes les échelles dans et hors de l'Union, mieux gérer les migrations sans que les pays de départ n'en récoltent que les effets négatifs à long terme, participer à la régulation de la mondialisation... Pour ce dernier aspect en particulier, il est devenu évident que peser très lourd est un gage de réussite lorsqu'on prétend convaincre le reste du monde d'utiliser certaines normes dans divers domaines (environnement, droit du travail, droits de l'homme, justice internationale, commerce des biens et des services, culture, commerce...). Même théoriquement forte d'un milliard d'habitants, l'Euroméditerranée pèse peu si elle ne fait pas l'effort de concevoir un début de charpente institutionnelle ou un socle partenarial plus consistant sur lequel pourraient s'appuyer des initiatives portées par les sociétés civiles ou éventuellement par les entreprises de l'UE et des voisinages. Sans ce socle, même a minima, il sera difficile voire impossible de traiter des problèmes communs pourtant urgents. Le jeu en vaut la chandelle : une étude de l'Ifri montre que l'UE ne pourra maintenir sa compétitivité à l'échelle mondiale qu'en renforçant ses liens avec ses voisins (Colombani, 2002). Pour avancer vers une plus grande régulation de la relation "UE - Voisinages", il faudra que l'Union envoie des messages forts en direction de ces pays pour indiquer qu'elle souhaite mettre en œuvre un partenariat moins dissymétrique, souvent mal vécu par les différents voisins, et qu'elle ne se voit plus seulement comme une forteresse retranchée derrière une frontière ultra-sécurisée. Dans ce dernier domaine, les nombreuses déclarations récurrentes d'officiels européens contre l'érection de frontières barrières et de nouvelles lignes de fracture ne convainquent pas les autorités politiques et les opinions publiques des pays proches. Organiser la proximité géographique et la complémentarité fonctionnelle des pays de ce vaste ensemble géographique UE Voisinages est aussi important pour les voisins eux-mêmes. Peuvent-ils continuer à subir la fuite de leurs cerveaux ? Peuvent-ils se résoudre à rester si peu attractifs pour les investisseurs étrangers? Doivent-ils compter sur leurs seules forces pour faire face à des problèmes aussi graves que la pénurie d'eau, l'exode rural et l'urbanisation accélérés, l'effort d'éducation et de formation, la concurrence commerciale d'autres économies, etc. ? En bref, l'échelle macrorégionale peut-elle contribuer efficacement à la délicate question de la gouvernance de la mondialisation?

\section{Sommaire de l'ouvrage}

Abordant ces nombreux problèmes, cet ouvrage apporte des éléments d'analyse pour mieux comprendre les relations entre l'UE et ses voisinages. Le premier objectif d'ensemble est de juger la qualité actuelle et la manière dont ces relations sont organisées. Le deuxième est de faire quelques recommandations pour mettre en œuvre une relation mieux régulée. La parole a été donnée à deux types d'auteurs : d'une part, 
des chercheurs et des universitaires qui contribuent à la construction d'un savoir académique avec des méthodes scientifiques bien établies ; d'autre part, des acteurs ou des professionnels de la relation «UE - Voisinages ». Ces « acteurs » sont des personnes impliquées dans l'action et qui produisent cette relation au jour le jour, ce qui n'enlève rien à la pertinence de leur analyse, au contraire. Aussi certains articles ne présententils pas les caractères classiques de la production scientifique. Mais il nous a semblé très utile et éclairant de croiser les regards pour susciter des réactions (voir à ce propos la contribution d'Élise Temple-Boyer).

Les articles de la première partie abordent la relation entre l'Union et l'ensemble de ses voisinages, sans privilégier telle ou telle région. Trois thèmes sont abordés. Dans sa contribution sur le thème de l'élargissement, Jean-François Drevet pose la question non tranchée des limites de ce processus, dans le temps et dans l'espace. Cette question est d'autant plus importante que, à ce jour, seule la perspective d'adhésion s'est révélée être un levier d'influence efficace de l'UE sur ses voisins en les contraignant à engager des réformes lourdes. Quiddans ce cas des pays proches qui ne seront jamais membres? L'UE est-elle condamnée à n'y être jamais vraiment entendue ? L'article de Nicolas Gaubert et Yann Richard se concentre sur la politique européenne de cohésion. Celle-ci souffre d'une évidente étroitesse de vue : en ne prenant pas assez en considération les interactions qui existent entre le territoire communautaire et les territoires des pays voisins, la Commission européenne ne réduit-elle pas l'efficacité de sa propre politique de cohésion? Les réformes réglementaires intervenues en 2007 apportent-elles une réponse adéquate à cette myopie politique? L'article de Catherine Wihtol de Wenden est consacré à la question migratoire. L'UE est le premier pôle migratoire du monde, nettement devant les États-Unis, mais elle ne se perçoit pas comme tel. Cet autre signe de myopie politique empêche l'UE d'avancer vers une gestion efficace et concertée des flux de migrants internationaux avec les pays voisins.

Les articles de la deuxième partie sont issus des travaux de l'Institut de Prospective Économique du Monde méditerranéen. L'objectif de l'IPEMed est de contribuer au passage des diagnostics à l'action, difficulté typiquement méditerranéenne qui fait l'objet du texte de Pierre Beckouche. Beaucoup de bonnes analyses, peu de réalisations concrètes : tel pourrait être le bilan de treize ans du processus de Barcelone, qui devait établir sur le flanc sud de l'Europe des relations de paix et de prospérité. Les textes rassemblés ici ne font pas le bilan de Barcelone, ils ne commentent pas plus les raisons du passage de l'UE à la politique de Voisinage. Ils se concentrent plutôt sur quelquesuns des enjeux sectoriels les plus délicats de l'Euroméditerranée, que la politique de Voisinage et l'Union pour la Méditerranée devront essayer de faire avancer: la question agricole d'abord, l'accès au marché du Nord pour les produits agricoles des pays du Sud étant une des causes de l'échec de la régulation internationale et en particulier du cycle de Doha. Jean-Louis Rastoin établit le diagnostic régional de la filière fruits et légumes, insiste sur la concurrence croissante des autres régions du monde. Il conclut sur la nécessité de valoriser des produits caractérisés en Méditerranée par un ancrage territorial fort. Christian Dubreuil en tire les conséquences politiques : l'échelle régionale peut être la bonne échelle pour trouver des régulations internationales innovantes. La question de l'eau ensuite, vaste question à laquelle le réchauffement climatique donne, en Méditerranée, une dimension potentiellement dramatique. Claude Martinand l'aborde sur le versant de la politique intégrée d'accès à l'eau et de l'assainissement. Il donne quelques clés pour faire 
progresser les partenariats Nord-Sud et notamment les partenariats public (Sud)-privé (Nord), tout juste balbutiants. La question du financement fait l'objet de la contribution de Guillaume Almeras et Abderrahmane Hadj Nacer. Ils nient que l'absence de moyens financiers soit une des causes du mal développement au Sud. Selon eux, au contraire, la caractéristique de la rive sud, c'est l'abondance d'argent, qu'il s'agisse d'épargne locale ou des transferts des immigrés installés sur la rive nord. Ils voient dans la canalisation des remises d'épargne une source potentielle déterminante pour le partenariat euroméditerranéen, qui n'aurait donc selon eux pas besoin de subventions européennes. Cela suppose de faire des échanges transméditerranéens et des modes de vie à cheval sur les deux rives, des éléments centraux du partenariat. Or pour le moment, rappellent Philippe Fargues et Hervé Le Bras, la mobilité transméditerranéenne est combattue par l'UE, les pays européens continuant à raisonner en termes de migrations alors que la réalité géographique est celle de mobilités croissantes. Ces contributions mettent l'accent sur des besoins de connaissance qui touchent à des aspects très concrets des secteurs d'activité et des territoires méditerranéens. Elles soulignent le besoin de couplage avec des décisions opérationnelles. C'est le positionnement de l'IPEMed, qui associe recherche et action, université et décision. D'ailleurs, l'idée d'Union pour la Méditerranée est née de cette prise de conscience qu'un type nouveau de mobilisation de la connaissance, davantage au service d'actions concrètes de développement, devrait s'ajouter aux procédures administratives classiques de l'Europe à l'égard de ses voisins du Sud.

La troisième partie conserve une approche régionale en s'ouvrant sur trois horizons (les Balkans, la Russie et la mer Méditerranée) et en explorant trois thèmes différents. L'article d'Amaël Cattaruzza analyse la montée en puissance de l'UE dans les Balkans avec lesquels elle entretient des relations de plus en plus intenses tout en encourageant un processus de rapprochement entre les États de cette région. L'action de l'Union, en créant des ensembles régionaux constamment remaniés, a eu des résultats ambigus. L'article d'Antoine Beyer explore le thème de l'intégration régionale Ouest-Est par le biais des infrastructures de transport en étudiant les modes de gestion de la différence d'écartement ferroviaire entre le système russe et le système standard européen. L'article d'Antoine Frémont montre que la Méditerranée reste une fracture en matière de transport maritime entre les deux rives et que cette plaine de transport demeure largement structurée en fonction des besoins de la rive nord. On est encore loin d'une gestion régionale, alors qu'une telle démarche pourrait avoir un impact positif sur l'ensemble de la région méditerranéenne.

\section{BIBLIOGRAPHIE}

Arbatova N., « Russie-UE après 2007 : le débat russe », Russie. NEI. Visions, n² 20, IFRI.

Beckouche P., 2008, Les régions Nord Sud. Euromed face à l'intégration des Amériques et de l'Asie orientale, Paris, Belin. 
Beckouche P., Grasland Cl., Didelon Cl., Richard Y., 2008, Europe in the World. Territorial Evidence and Visions, Paris, Luxembourg, Espon Programme, Université Paris 1, Université Paris 7, CNRS, Diact.

Beckouche P., Richard Y., à paraître en 2008, Atlas d'une nouvelle Europe. L'Union européenne et ses voisins : vers une région mondiale? Paris, Autrement.

Bordatchev T., 2007, « Représenter les intérêts privés pour renforcer la confiance entre la Russie et l'Union » in T. Gomart (dir.), La Russie. Enjeux internationaux et intérieurs, Politique étrangère, Paris, Ifri, Armand Colin, p. 51-62.

Broadman H., 2005, From Desintegration to Reintegration. Eastern Europe and the Former Soviet Union in the International Trade, World Bank.

Colombani Ph., 2002, Le commerce mondial au XXI ${ }^{e}$ siècle, Paris, IFRI.

Deblock Ch., Regnault, H. (dir.), 2006, Nord-Sud : la reconnexion périphérique, Montréal, Éditions Athéna.

Didelon Cl., Grasland, Cl., 2007, Europe in the World, rapport remis à la Commission européenne dans le cadre du programme Orate (Espon programme, project Espon 3.4.1). Disponible en intégralité sur le site Internet du programme Orate : www.espon.eu

Didelon Cl., Grasland, Cl., Richard, Y., à paraître en 2008, Atlas de l'Europe dans le monde, Paris, Reclus, La Documentation française.

Dollfus O., 1994, L'espace monde, Paris, Economica.

Dollfus O., 1999, La mondialisation, Paris, Presses de Sciences Po.

Dressler W. (dir.), à paraître en 2008, Eurasie, espace mythique ou réalité en construction ? Bruxelles, Bruylant.

Frankel J.A. (dir.), 1998, The regionalization of World Economy, Chicago, University of Chicago Press. Gomart T., 2007, « Politique étrangère russe : l'étrange inconstance », dans T. Gomart (dir.), La Russie. Enjeux internationaux et intérieurs, Politique étrangère, Paris, Ifri, Armand Colin, p. 191-206. Nicolas F., 2003, « À l'heure de la mondialisation. Mondialisation et intégration régionale, des dynamiques complémentaires », Cahiers français, $n^{\circ} 317, \mathrm{p}$ 59-63.

Philippart E., 2003, « The Euro-Mediterranean Partnership : Unique Features, First Results and Future Challenges », Center For European Policy Studies, Working Paper ${ }^{\circ} 10$.

Regnault H., « Nord et sud en Méditerranée : de la coopération à la confrontation, de la divergence à la convergence ?", Région et Développement, nº 19, p. 7-17.

Richard Y., Zanin C., 2007, « La Russie et l'Europe : une intégration économique encore à venir? ", Cybergéo.

Siroën J.-M., 2000, La régionalisation de l'économie mondiale, Paris, La Découverte.

Smith K., 2005, « The EU and Central and Eastern Europe : The Absence of Interregionalism, European Integration $", \mathrm{n}^{\circ}$ 3, p. 348-349.

Veltz P., 1996, Mondialisation, villes et territoires. L'économie d'archipel, Paris, PUF.

Wallerstein I., 1979, The Capitalist World Economy, Cambridge, Cambridge University Press.

Wallerstein I., 2002, Le capitalisme historique, Paris, La Découverte. Wallerstein I., 2006, Comprendre le monde, Paris, La Découverte. 


\section{NOTES}

1. Conseil de l'Union européenne, Bruxelles, 29 janvier 2003, Conseil européen de Copenhague, 12 et 13 décembre 2002, Conclusion de la Présidence, p. 7 et 8.

2. Le Conseil des affaires générales et des relations extérieures du 24 février 2003 parle par exemple de l'initiative sur le «nouveau voisinage " («new neighbourhood») qui avait été ellemême lancée lors d'un précédent Conseil en septembre 2002. Mais dans ce dernier cas, l'acception géographique n'était pas la même puisque Chris Patten s'était exprimé lors de ce conseil sur les relations de la future UE élargie avec les nouveaux voisins de l'Est. Le terme retenu n'était pas «new neighbourhood» mais "new neighbours » et se limitait à l'ensemble Biélorussie, Moldavie, Ukraine. Cf. General Affairs and External Relations, External Relations, Brussels, 30 septembre 2002.

3. General Affairs and External Relations, External Relations, Brussels, 18 mars 2003, p. 6.

4. Communication de la Commission au Conseil et au Parlement européen, L'Europe élargie Voisinage : un nouveau cadre pour les relations avec nos voisins de l'est et du sud, Bruxelles, 11mars 2003, COM (2003) 104 final. Les pays concernés par l'expression voisinage (au singulier) dans cette communication étaient les NEI occidentaux (Biélorussie, Moldavie, Ukraine), la Russie, les pays riverains de la Méditerranée.

5. Sur ce point, lire le Conseil affaires générales et relations extérieures, 14 juin 2004, p. 11-13.

6. C'est une des idées développées par la communication de la Commission «Relative au renforcement de la politique européenne de voisinage ", Bruxelles, 14 décembre 2006, COM (2006) 726 final. Le texte propose de développer les relations avec certains « voisins des voisins » de l'Union européenne, prenant acte ainsi de l'existence de relations actuelles ou potentielles importantes pour l'UE.

\section{INDEX}

Mots-clés : globalisation, démographie, voisinage, régionalisation, échanges commerciaux Index géographique : Europe

\section{AUTEUR}

YANN RICHARD

UMR Ladyss, université Paris 1 Panthéon Sorbonne 